\title{
Natural attack rate of influenza in unvaccinated children and adults: a meta-regression analysis
}

\author{
Kavisha Jayasundara ${ }^{1 *}$, Charlene Soobiah ${ }^{2}$, Edward Thommes ${ }^{1,3}$, Andrea C Tricco ${ }^{2,4}$ and Ayman Chit ${ }^{1,5}$
}

\begin{abstract}
Background: The natural (i.e. unvaccinated population) attack rate of an infectious disease is an important parameter required for understanding disease transmission. As such, it is an input parameter in infectious disease mathematical models. Influenza is an infectious disease that poses a major health concern worldwide and the natural attack rate of this disease is crucial in determining the effectiveness and cost-effectiveness of public health interventions and informing surveillance program design. We estimated age-stratified, strain-specific natural attack rates of laboratory-confirmed influenza in unvaccinated individuals.
\end{abstract}

Methods: Utilizing an existing systematic review, we calculated the attack rates in the trial placebo arms using a random effects model and a meta-regression analysis (GSK study identifier: 117102).

Results: This post-hoc analysis included 34 RCTs (Randomized Control Trials) contributing to 47 influenza seasons from 1970 to 2009. Meta-regression analyses showed that age and type of influenza were important covariates. The attack rates (95\% Cl (Confidence Interval)) in adults for all influenza, type A and type B were $3.50 \%(2.30 \%, 4.60 \%)$, $2.32 \%(1.47 \%, 3.17 \%)$ and $0.59 \%(0.28 \%, 0.91 \%)$ respectively. For children, they were $15.20 \%(11.40 \%, 18.90 \%), 12.27 \%$ $(8.56 \%, 15.97 \%)$ and $5.50 \%(3.49 \%, 7.51 \%)$ respectively.

Conclusions: This analysis demonstrated that unvaccinated children have considerably higher exposure risk than adults and influenza A can cause more disease than influenza B. Moreover, a higher ratio of influenza B:A in children than adults was observed. This study provides a new, stratified and up to-date natural attack rates that can be used in influenza infectious disease models and are consistent with previous published work in the field.

Keywords: Influenza A, Influenza B, Natural attack rate, Healthy individuals, Systematic review

\section{Background}

Infectious diseases are a major cause of morbidity and mortality worldwide [1]. The main focus for research on infectious diseases is to improve disease control and ultimately eradicate the infection from the population [2]. Mathematical models of infectious diseases are important for studying the impact of various interventions on disease outcome. Such investigations allow the implementation of targeted control measures, optimizing the use of limited resources [2].

The validity of a model is highly dependent on the validity of the input parameters. These models require information on parameters describing the population under study, disease transmission as well as parameters

\footnotetext{
* Correspondence: kavisha.s.jayasundara@gsk.com

'GSK Inc, 7333 Mississauga Road North, Mississauga, ON L5N 6L4, Canada Full list of author information is available at the end of the article
}

describing the health outcomes of infection. Lack of reliable data complicates the development of models and new data needs to be collected or analyzed or the model itself needs to be fitted to available data to estimate input parameters [3].

Influenza is an infectious disease that poses a major health concern all over the world. The total economic burden of influenza in the United States has been estimated as $\$ 87.1$ billion [4]. Developing mathematical models for influenza can be used to determine how to mitigate disease. The attack rate of the influenza virus is crucial for these models and a difficult parameter to obtain.

Generally, influenza epidemic infection rates are obtained from influenza-like illness (ILI), surveillance data and serological surveys [5]. In a static (e.g. decision tree) model, the attack rate is often used directly as an input parameter, whereas in a dynamic transmission model, it

Ciomed Central

(c) 2014 Jayasundara et al.; licensee BioMed Central Ltd. This is an Open Access article distributed under the terms of the Creative Commons Attribution License (http://creativecommons.org/licenses/by/4.0), which permits unrestricted use, distribution, and reproduction in any medium, provided the original work is properly credited. The Creative Commons Public Domain Dedication waiver (http://creativecommons.org/publicdomain/zero/1.0/) applies to the data made available in this article, unless otherwise stated. 
is used to calibrate, or calculate directly, the force of infection. Whether static or dynamic, it is most preferable to use the "natural attack rate", that is, the attack rate within the unvaccinated portion of the population. This is a more "universal" quantity, since it is not as strongly dependent on the population vaccine uptake, though it is still coupled through herd immunity.

Turner et al. used age-specific attack rates of influenza in an economic evaluation based on rates derived from placebo-controlled trials of influenza vaccines and antivirals [6]. The incidence of symptomatic laboratory-confirmed influenza cases in each placebo arm was used to estimate the corresponding attack rates for each subgroup using a random effects model. Overall, the attack rate was 6.55\% (range: 0 to $20 \%$ ) for adults and $19.21 \%$ (range: 10 to $35 \%$ ) for children. All trials except one were in the US and spanned eight influenza epidemics during the period 1984 through 1997-1998. These attack rates were not adjusted for confounders and also were not stratified by strain of influenza.

The objective of this study was to estimate the attack rate of influenza stratified by both age and influenza strain (A and B) for unvaccinated individuals measured by laboratory-confirmed PCR (Polymerase Chain Reaction) and/or culture.

\section{Methods}

This study (GSK study identifier: 117102) is a post-hoc analysis of a published systematic review [7]. The full methods have been published $[7,8]$ and the protocol was registered in the PROSPERO database (CRD42012001926).

\section{Eligibility criteria}

Healthy children, adults or the elderly were chosen as the population of interest. All vaccine types were included: trivalent influenza vaccines (TIV), live-attenuated influenza vaccines (LAIV), and bivalent vaccines. Randomized controlled trials (RCTs) or quasi-RCTs, written in English, comparing influenza vaccine with placebo and reporting data on the primary outcomes were included. The primary outcome used was laboratory-confirmed influenza by PCR and/or culture [7]. The secondary outcome included haemagluttination inhibition (HAI) assay alone or in combination with PCR and/or culture.

\section{Information sources and search}

Medical subject headings (MeSH) and text words related to influenza vaccination were used in the literature search strategies. MEDLINE, EMBASE and Cochrane Central Register of Controlled Trials were searched by an experienced librarian. This was supplemented by searching for trial protocols through metaRegister (http://www. controlled-trials.com/mrct/), which allows the searching of multiple trial protocol registries simultaneously. The reference lists of included studies and relevant reviews were scanned to ensure all studies were captured. Authors of studies were contacted for additional information, as necessary.

\section{Study selection process}

Online proprietary systematic review software Synthesi.SR (http://knowledgetranslation.ca/sysrev/login.php), available through the Li Ka Shing Knowledge Institute of St. Michael's hospital was used to screen citations and fulltext articles that were identified through the literature search. After a team calibration exercise, the Synthesi.SR program was used to screen citations and full-text articles. Each citation and full-text article was screened by two reviewers independently.

\section{Data items}

The study characteristics abstracted were as follows: year of conduct, sample size, country of conduct, setting, viral strain(s) detected (strain A or B), age of study population (mean and standard deviation), infection detection method (PCR, viral culture, HAI), number of confirmed influenza infections in the placebo arm and the total number of patients in the placebo arm.

\section{Synthesis of included studies}

The data was first summarized descriptively. The natural attack rate was computed using the following formula: number of influenza cases/number of subjects in the placebo arm $\times 100 \%$, as per the methods in Turner et al. [6]. The overall natural attack rate of influenza for all unvaccinated individuals was derived using a random effects model which allows for within and between study variance [6]. Separate analyses were conducted for children and adults (children: $<18$ years, Adults: $\geq 18$ years), strain of influenza confirmed by laboratory testing (strain A or B), the type of test used for laboratory confirmation (PCR and/or culture vs. HAI testing alone or in combination with PCR or culture). Time trends for attack rates were observed through forest plots.

\section{Meta-regression analysis}

Important covariates that would influence the natural attack rate of influenza were identified a priori. They were age group, geographical region where the study was conducted, type of laboratory-confirmed influenza detected (all influenza, strain A and strain B), type of test used to confirm infection (PCR and/or culture vs. HAI testing alone or in combination with PCR or culture) and the year of influenza season. Univariate analyses were conducted on these covariates to determine their statistical significance. Based on those results, meta-regression analysis was performed on statistically significant covariates. 
It is not always preferred to run all possible covariates in a meta-regression model as it would reduce statistical power of the results and lead to larger confidence intervals. The total number of covariates was constrained so that there were enough studies per outcome [9]. Additionally, statistical heterogeneity was tested using the $\mathrm{I}^{2}$ statistic [10]. If high heterogeneity was observed, meta-regression analysis was conducted. All statistical analyses were conducted using $R$ version 2.1.

\section{Results}

The systematic review informing this analysis yielded 1356 citations [7]. A total of 34 RCTs and 1 companion report fulfilled the inclusion criteria (Figure 1). These 34 trials contributed to 47 influenza seasons from 1970 to 2009 including 94,821 participants. Reasons for exclusion, study characteristics, and patient characteristics are reported in detail elsewhere [7]. The trials were conducted in North America (22/34), Europe (4/34), Australia (1/34) and a mixture of multi-site trials in North America, Europe, South America, Asia (7/34).

From the 47 influenza seasons identified, 37 reported on the primary outcome (laboratory confirmed influenza by PCR and/or culture) and all 47 reported on the secondary outcome (laboratory confirmed influenza by HAI assay alone or in combination with PCR and/or culture).
From the 37 influenza seasons that reported on the primary outcome, 34 reported on influenza A and 35 reported on influenza B. From the 47 influenza seasons that reported on the secondary outcome, 41 reported on influenza A and 42 reported on influenza B. Attack rates were calculated for all age groups separated by type of influenza using a random effects model (Table 1). All results are reported in the following format: point estimate, 95\% confidence interval [CI, (lower level, upper level)]. For the primary outcome, the overall attack rate was $7.86 \%(5.48 \%, 10.23 \%)$ for all influenza, $5.68 \%(3.71 \%$, 7.65\%) for strain A and 1.87\% (1.02\%, 2.72\%) for strain B. For the secondary outcome, an attack rate of $9.84 \%$ $(7.06 \%, 12.62 \%)$ was observed for all strains, $6.96 \%$ (4.49\%, 9.42\%) for strain A and 2.57\% (1.45\%, 3.70\%) for strain B. The results of the random effects model also demonstrated high statistical heterogeneity the high $\mathrm{I}^{2}$ values.

Meta-regression analysis was conducted to reduce the heterogeneity associated with the attack rate calculations. Age was considered an important covariate in natural attack rate. In order to confirm this with our data, a meta-regression analysis was conducted with age stratifications. With the available data from the studies from the systematic review, we were only able to separate ages into two categories: adults and children. Limited number of trials reported on the elderly population alone and

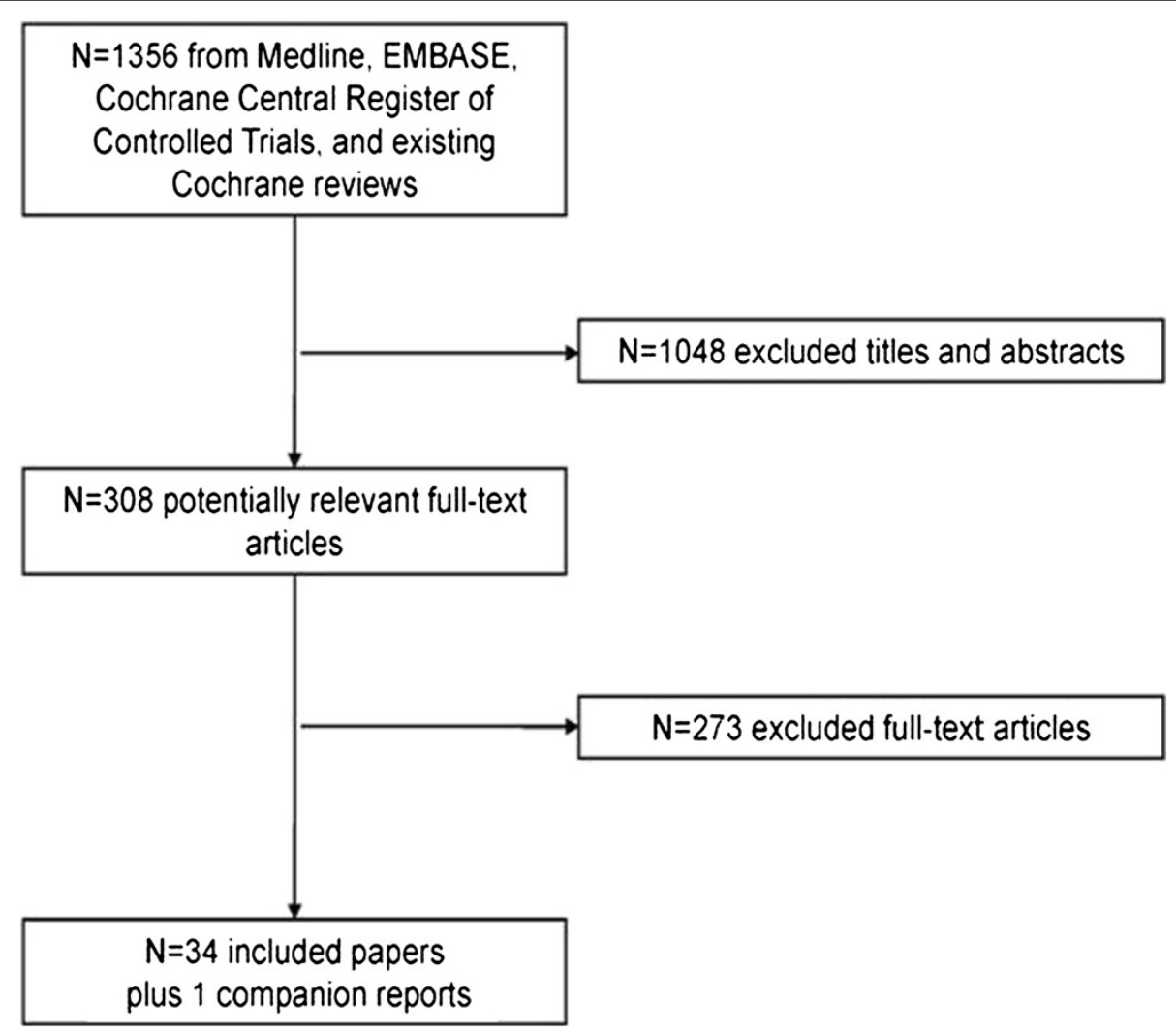

Figure 1 Study flow chart. 
Table 1 Meta-analysis results comparing primary outcome (RT-PCR and/or culture) with secondary outcome (RT-PCR and/or culture and/or HAl assay) for all ages

\begin{tabular}{lllll}
\hline Type of influenza & \# of influenza seasons & $\begin{array}{l}\text { Sample size } \\
\text { (placebo group) }\end{array}$ & $\begin{array}{l}\text { \# of influenza cases } \\
\text { (placebo group) }\end{array}$ & $\begin{array}{l}\text { Natural attack rate \% } \\
\text { (lower 95\% Cl, upper 95\% Cl) }\end{array}$ \\
\hline $\begin{array}{llll}\text { Primary outcome (PCR and/or viral culture) } \\
\text { All }\end{array}$ & 39115 & 1774 & $7.86(5.48,10.23)$ \\
Influenza A & 37 & 38360 & 1303 & $5.68(3.71,7.65)$ \\
Influenza B & 34 & 38854 & 491 & $1.87(1.02,2.72)$ \\
Secondary outcome (PCR and/or viral culture and/or HAl assay) & & & $99.86 \%$ \\
All & 35 & 41212 & 2055 & $9.84(7.06,12.62)$ \\
Influenza A & 47 & 40074 & 1505 & $6.96(4.49,9.42)$ \\
Influenza B & 41 & 40568 & 536 & $2.57(1.45,3.70)$
\end{tabular}

Abbreviations: $C l$ confidence interval, $H A l$ haemagglutination inhibition assay, $R T-P C R$ reverse transcription polymerase chain reaction.

most of them did not report the primary outcome. It was difficult to use geography as a covariate since many trials were multi-national in nature. Due to this limitation, we did not include geography as a covariate in the meta-regression.

Data was separated by primary and secondary outcome and type of influenza to determine whether adults and children experience different attack rates. The results of the meta-regression analysis are shown in Table 2. For all influenza, adults exhibit an attack rate of $4.4 \%$ and children experienced an attack rate that was $11 \%$ higher than adults. For influenza A, adults exhibited an attack rate of $3.4 \%$ and children experienced a higher attack rate than adults by $8.8 \%$. For influenza B, adults showed an attack rate of $1.1 \%$ and children showed an attack rate that was 4.4\% higher than that of adults. All analyses were statistically significant and suggested that a subgroup analysis by age group is required for the primary outcome for all influenza, influenza A and B.

When a similar meta-regression was conducted on the broader secondary outcome, higher attack rates were observed overall. Results can be seen in Table 3. Adults, on average, exhibited at attack rate of $5.8 \%$ for all influenza with children experiencing a rate that is $13.9 \%$ higher than that of adults. For influenza A, adults experienced

Table 2 Meta-regression for primary outcome

\begin{tabular}{llll}
\hline & Estimate & Standard error & P-value \\
\hline Influenza A and B & & & \\
(Intercept) & 0.044 & 0.012 & 0.0012 \\
$\begin{array}{l}\text { Children vs. Adults } \\
\text { Influenza A }\end{array}$ & 0.110 & 0.019 & $<0.0001$ \\
$\begin{array}{l}\text { Intercept) } \\
\text { Children vs. Adults }\end{array}$ & 0.034 & 0.011 & 0.005 \\
Influenza B & & 0.018 & $<0.0001$ \\
(Intercept) & 0.011 & 0.0055 & \\
Children vs. Adults & 0.044 & 0.009 & 0.046 \\
\hline
\end{tabular}

an attack rate of $4.2 \%$ with children having an attack rate $7.9 \%$ higher than adults. Attack rate of influenza B was lower than that of influenza A, with adults exhibiting an attack rate of $1.4 \%$ and children having an attack rate that is $5.6 \%$ higher than that of adults. These analyses were all statistically significant. The trends of higher attack rates for children than adults, and higher attack rates for strain A than strain B were also present when analyzing the secondary outcome.

Since we confirmed that age and type of influenza were important covariates for natural attack rates, we then conducted subgroup analysis by age group (adults and children) and type of influenza (all, type A and type B) for the primary outcome (Table 4). The overall attack rate for adults for all influenza was $3.50 \%(2.30 \%, 4.60 \%)$ and when separated by type, $2.32 \%(1.47 \%, 3.17 \%)$ for influenza A and $0.59 \%(0.28 \%, 0.91 \%)$ for influenza B. For children, the overall attack rate was $15.20 \%(11.40 \%, 18.90 \%)$ for all influenza. When stratified by type, $12.27 \%(8.56 \%, 15.97 \%)$ for influenza A and 5.50\% (3.49\%, 7.51\%) for influenza B. Trials that reported outcomes specifically on adults and children were included in this analysis and trials that reported on mixed populations were excluded. Even with the sub group analysis by age and type of influenza we still observed high $\mathrm{I}^{2}$ values.

Table 3 Meta-regression for secondary outcome

\begin{tabular}{|c|c|c|c|}
\hline & Estimate & Standard error & P-value \\
\hline \multicolumn{4}{|l|}{ Influenza $A$ and $B$} \\
\hline (Intercept) & 0.058 & 0.018 & 0.0024 \\
\hline Children vs. Adults & 0.139 & 0.028 & $<0.0001$ \\
\hline \multicolumn{4}{|l|}{ Influenza A } \\
\hline (Intercept) & 0.042 & 0.018 & 0.0225 \\
\hline Children vs. Adults & 0.079 & 0.0279 & 0.0074 \\
\hline \multicolumn{4}{|l|}{ Influenza B } \\
\hline (Intercept) & 0.014 & 0.015 & 0.375 \\
\hline Children vs. Adults & 0.056 & 0.024 & 0.025 \\
\hline
\end{tabular}


Table 4 Meta-analysis on primary outcome for adults and children

\begin{tabular}{lllll}
\hline Type of influenza & \# Influenza seasons & $\begin{array}{l}\text { Sample size } \\
\text { (placebo group) }\end{array}$ & $\begin{array}{l}\text { \# Influenza cases } \\
\text { (placebo group) }\end{array}$ & $\begin{array}{l}\text { Natural attack rate \% } \\
\text { (lower 95\% Cl, upper 95\% Cl) }\end{array}$ \\
\hline Adults & & & & $\mathbf{I}^{\mathbf{2}}$ \\
All & 19 & 28748 & 627 & $3.50(2.30,4.60)$ \\
Influenza A & 19 & 28748 & 482 & $2.32(1.47,3.17)$ \\
Influenza B & 19 & 28748 & 150 & $0.59(0.28,0.91)$ \\
Children & & & & \\
All & 13 & 6187 & 1040 & $15.20(11.40,18.90)$ \\
Influenza A & 10 & 5432 & 714 & $12.27(8.56,15.97)$ \\
Influenza B & 11 & 5926 & 341 & $5.50(3.49,7.51)$
\end{tabular}

Abbreviations: $\mathrm{Cl}$ confidence interval.

The forest plot for influenza $\mathrm{A}$ infections in children and adults for the primary outcome can be seen in Figure $2 \mathrm{~A}$ and $\mathrm{B}$ respectively. The forest plot for influenza B for children and adults can be seen in Figure 3A and $\mathrm{B}$ respectively. Generally, no time trends were observed in the attack rate for adults or children for each type of influenza.

\section{Discussion}

This study estimated the natural attack rate of influenza for unvaccinated children and adults using a post-hoc analysis of a previously conducted systematic review. This is the best method to obtain such attack rates since currently existing methods to obtain this data have a number of limitations. First, estimating attack rates from surveillance data provides attack rates as an aggregate of vaccinated and unvaccinated individuals and not attack rates that are specific to unvaccinated individuals that are presented here. Second, extrapolating from surveillance data also necessitates estimating rates of primary care consultations among influenza cases [5]. Third, even if there is an active surveillance system looking for influenza, surveillance systems will be limited by under-reporting bias [11]. Finally, it's difficult to determine the size of the population under surveillance which makes estimating rates of infection difficult as there is uncertainty around the denominator of the ratio [11].

Although yearly influenza epidemics can seriously affect all age groups, children younger than two years of age and adults over 65 exhibit higher rates of disease [12]. Further, contact pattern studies suggest that children are more vulnerable to infection due to their relatively more frequent and longer intimate contacts with members of their age group [13]. Therefore, age is believed to be important in the natural attack rate of influenza. Geography dictates contact patterns and seasonality which is known to be a driver in severity of influenza [14]. Outbreaks of seasonal influenza follow largely predictable seasonal patterns and in temperate climate zones seasonal influenza epidemic lasts from six to ten weeks and in tropical zones seasonal patterns are less pronounced with more than one peak of infection [15]. Thus, geography is also an important consideration with regards to natural attack rate. With respect to the two main types of influenza in circulation, influenza A contributes to greater annual epidemics and infrequent yet more-devastating pandemics than influenza B [16]. Therefore, it's important to distinguish between these two types of influenza types. As the sensitivity of various laboratory methods for detecting influenza can yield different results, this was also included as an important covariate. Since yearly differences in influenza are seen from season to season, the year of study conduct was also considered an important covariate. Therefore, age group, geographical region, type of laboratory-confirmed influenza detected, type of test used to confirm detection and the year of influenza season were all considered important covariates of natural attack rate in our study.

Meta-regression analyses conducted here showed that age group and type of influenza are important covariates in natural attack rates. For adults, an attack rate of 3.50\% $(2.30 \%, 4.60 \%)$ was observed for all influenza strains. When stratified by type, an attack rate of $2.32 \%(1.47 \%$, 3.17\%) was observed for influenza A and a rate of $0.59 \%$ $(0.28 \%, 0.91 \%)$ was observed for strain B. For children, the overall attack rate was $15.20 \%(11.40 \%, 18.90 \%)$ and when stratified, a rate of $12.27 \%(8.56 \%, 15.97 \%)$ was seen for strain A and $5.50 \%(3.49 \%, 7.51 \%)$ was seen for strain B. The weighted sum of the stratified rates may not add up to the aggregate rates as some studies reported results for both strains of influenza. Including HAI testing increased the attack rate for all age groups and all types of influenza. No general trends with time were observed for children and adults for the primary outcome.

This study provides new, up to-date estimates of the attack rate of influenza in children and adults separated by type of influenza. Generally, our overall attack rates for adults and children were lower than the attack rates observed by Turner et al. [6]. The Turner analysis included trials covering only 8 influenza seasons from 1984 to 1998 while our analysis covered 47 influenza 


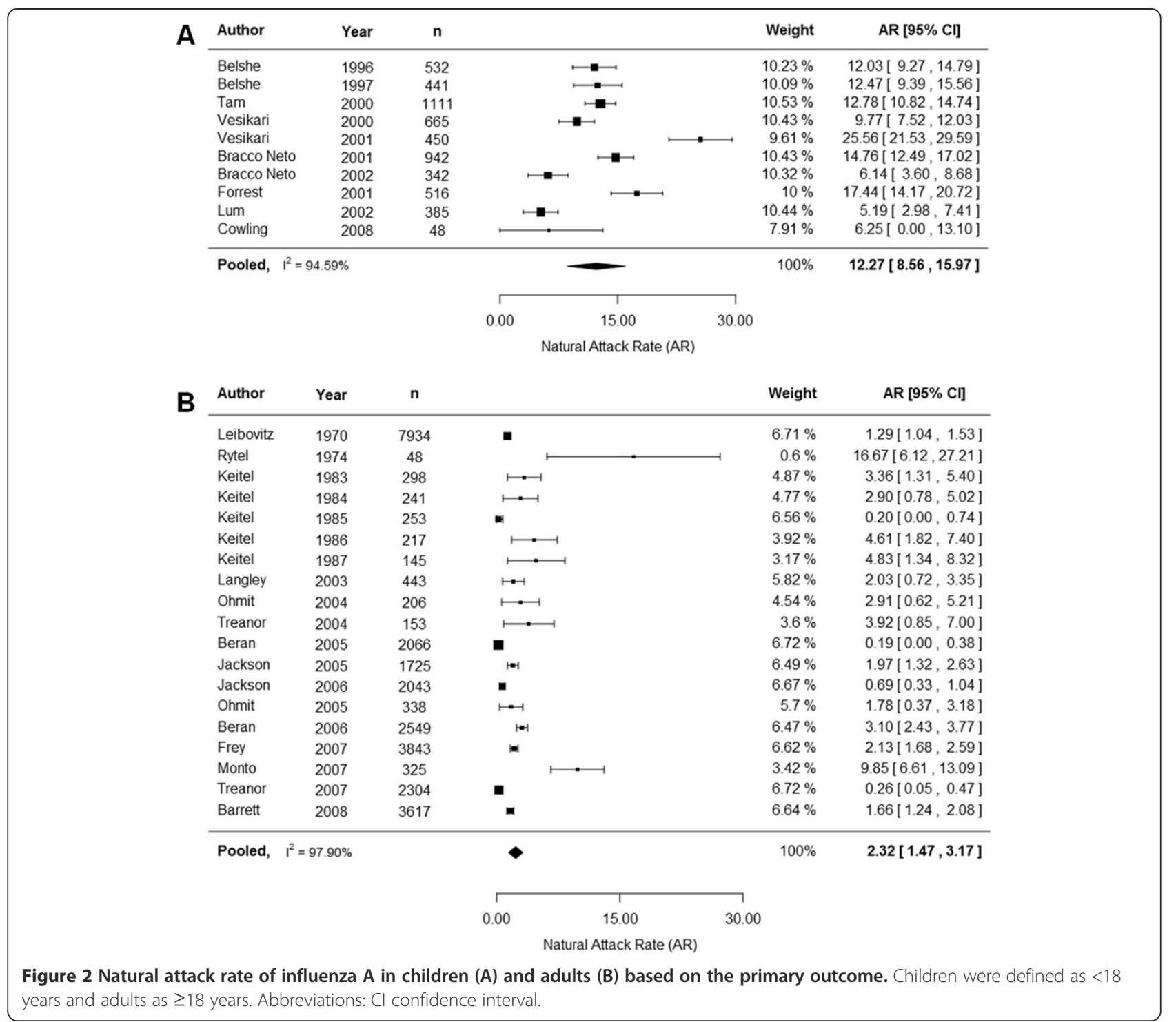

seasons from 1970 to 2009. The primary outcome of our studies was PCR and/or culture but the studies used in the Turner analysis did not include PCR as a method of detection. PCR is considered the most reliable diagnostic test for clinical practice with a higher accuracy of detection [17]. Using culture as a method of detection may miss influenza cases and the use of HAI testing may lead to biased results [17]. This may explain why the attack rates observed in the Turner analysis are higher than the results we have obtained $(6.55 \%$ for adults versus $3.57 \%$ and $19.21 \%$ for children versus $14.22 \%)$. Our secondary outcome, which included HAI testing, also showed an increase in attack rates for all age groups and types of influenza which supports the notion that higher attack rates are observed when HAI testing is used as a method of detection. Furthermore, the definition of children used by our study and by the
Turner study is quite different. In our study, children were classified as $<18$ years while the Turner classified them as $<12$ years. This may also attribute to the differences seen in attack rates when comparing between studies. Influenza B is most prominent among older children and young adults [18]. In line with this, our attack rates for children exhibited a higher B:A ratio than for adults. The B:A attack rate ratio for children was calculated to be 0.45 while the ratio for adults was 0.25 . This further exemplifies that using this method to calculate the natural attack rate does in fact reflect what is observed in real life settings for seasonal influenza [18]. The strengths of our analysis over the Turner analysis include the use of many trials covering more influenza seasons, using a more accurate method of detection (RT-PCR (Reverse Transcription Polymerase Chain Reaction) and/or culture), adjusting for confounders that 


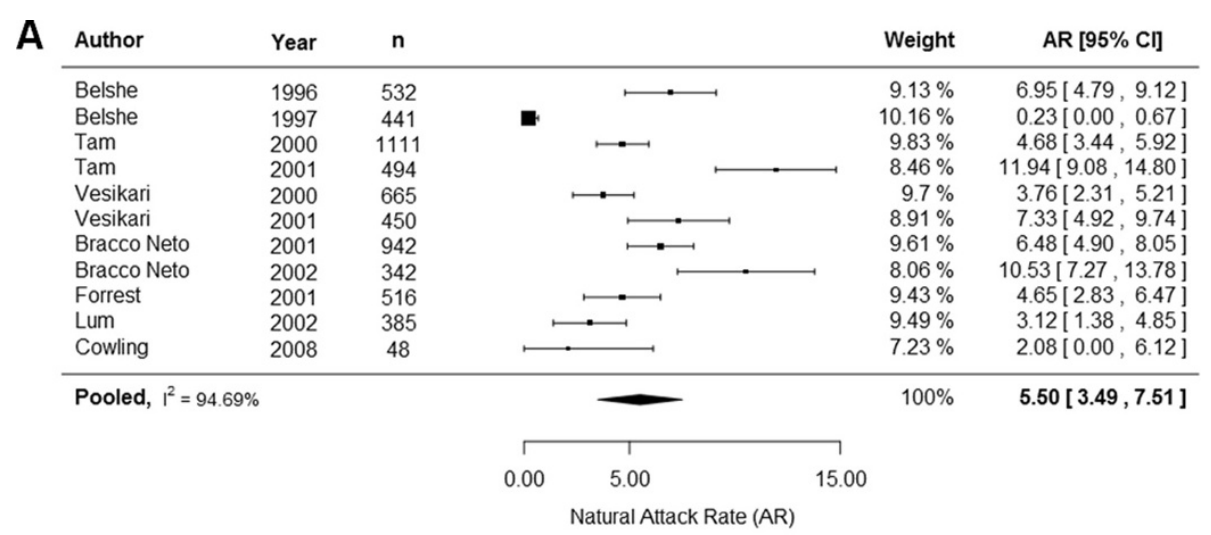

\begin{tabular}{|c|c|c|c|c|c|}
\hline Author & Year & $\mathrm{n}$ & & Weight & $\operatorname{AR}[95 \% \mathrm{Cl}]$ \\
\hline Leibovitz & 1970 & 7934 & घ & $7.99 \%$ & $0.01[0.00,0.02]$ \\
\hline Rytel & 1974 & 48 & $\longmapsto$ & $1.07 \%$ & $1.02[0.00,3.83]$ \\
\hline Keitel & 1983 & 298 & $\longmapsto$ & $2.13 \%$ & $2.68[0.85,4.52]$ \\
\hline Keitel & 1984 & 241 & $\mapsto-1$ & $6.3 \%$ & $0.21[0.00,0.78]$ \\
\hline Keitel & 1985 & 253 & $\longmapsto$ & $0.95 \%$ & $6.32[3.32,9.32]$ \\
\hline Keitel & 1986 & 217 & $10-1$ & $6.01 \%$ & $0.23[0.00,0.86]$ \\
\hline Keitel & 1987 & 145 & $\longmapsto$ & $1.48 \%$ & $2.07[0.00,4.39]$ \\
\hline Langley & 2003 & 443 & $H-1$ & $7.4 \%$ & $0.11[0.00,0.42]$ \\
\hline Ohmit & 2004 & 206 & $\neg$ & $1.5 \%$ & $2.91[0.62,5.21]$ \\
\hline Treanor & 2004 & 153 & $\longmapsto$ & $2.19 \%$ & $1.31[0.00,3.11]$ \\
\hline Beran & 2005 & 2066 & re-1 & $7.25 \%$ & $0.68[0.32,1.03]$ \\
\hline Jackson & 2005 & 1725 & rot & $7.67 \%$ & $0.23[0.00,0.46]$ \\
\hline Jackson & 2006 & 2043 & ret & $7.54 \%$ & $0.39[0.12,0.66]$ \\
\hline Ohmit & 2005 & 338 & $1 \cdot-1$ & $6.27 \%$ & $0.30[0.00,0.87]$ \\
\hline Beran & 2006 & 2549 & $m$ & $7.88 \%$ & $0.12[0.00,0.25]$ \\
\hline Frey & 2007 & 3843 & $\mapsto-1$ & $7.09 \%$ & $1.59[1.19,1.98]$ \\
\hline Monto & 2007 & 325 & $\longmapsto$ & $3.67 \%$ & $1.23[0.03,2.43]$ \\
\hline Treanor & 2007 & 2304 & - & $7.97 \%$ & $0.02[0.00,0.08]$ \\
\hline Barrett & 2008 & 3617 & tor & $7.63 \%$ & $0.55[0.31,0.79]$ \\
\hline \multirow[t]{3}{*}{ Pooled, } & & & $\bullet$ & $100 \%$ & $0.59[0.28,0.91]$ \\
\hline & & & $T$ & $\neg$ & \\
\hline & & & 5.00 & 10.00 & \\
\hline
\end{tabular}

Figure 3 Natural attack rate of influenza B in children (A) and adults (B) based on the primary outcome. Children were defined as $<18$ years and adults as $\geq 18$ years. Abbreviations: $\mathrm{Cl}$ confidence interval.

affect the natural attack rate and providing strain-specific natural attack rates for influenza.

Like any meta-analysis, our study has several limitations. First, although we identified three RCTs reporting data in the senior population, we were not able to derive an attack rate for this population. Two out of the three studies did not report on our primary outcome of interest and the third study reported an attack rate of $0 \%$. Second, we faced an inability to calculate geographyspecific attack rates. Although it was decided $a$ priori that geography is an important covariate, the data did not support such an analysis. Although most of the trials were conducted in North America, newer trials were multicentre in nature spanning over more than one continent, making a geography-specific analysis difficult. Third, the attack rates that are calculated here are attenuated by community herd effects through vaccinated people in the population. For countries with a low background vaccination rate, natural attack rates might be higher than what's reported here. As most of the studies in this analysis were conducted in North America, the attack rates obtained here would reflect the effects of herd immunity observe through targeted vaccine coverage programs that are used in this region. Furthermore, the attack rates observed in this study are laboratory confirmed. A study by GlatmanFreedman et al. have shown that in general, laboratory confirmed attack rates are higher than clinical attack rates which is an important consideration when using these rates as model inputs [19]. Another limitation of this study is that $\mathrm{H} 1 \mathrm{~N} 1$ and $\mathrm{H} 3 \mathrm{~N} 2$ strains were agglomerated as strain A for analysis. The attack rates for these two types for strain A are different from each other and thus lead to 
different health outcomes. Also, the severity of the influenza strains are known to be in the order of H3N2 > B $>$ H1N1 [18]. Therefore, one must be cautious when applying the agglomerated attack rate presented here for strain A to reflect $\mathrm{H} 3 \mathrm{~N} 2$ or $\mathrm{H} 1 \mathrm{~N} 1$ specifically. Although we identified one study that focused on the H1N1 pandemic flu, Talaat et al. [20], it reported on adults and elderly population together and therefore was not included in the meta-regression of attack rate for adults. Lastly, as this study is based on the results of an already conducted systematic review, it is inherently limited by the results of the systematic review. The systematic review only focused on RCTs of vaccines and not antivirals (as was done for the Turner et al. analysis). If the focus of the systematic review was expanded to include antivirals, then a large sample size would have been available for the attack rate calculations and perhaps lead to reduced uncertainty of the estimates that were derived from the analysis.

Influenza models are important tools in public health decision-making, and natural attack rate of influenza is a vital ingredient for such models, as either a direct input parameter or a calibration target. It is imperative that robust data are used to inform these models so that they can better predict disease transmission and also resource allocation that is required to reduce the burden of illness. The sophistication of mathematical models of disease transmission has increased in the last decade [21,22]; accordingly, the need for valid, precise and accurate model inputs, such as natural attack rate, is greater than ever. The over- or under-estimation of these parameters can have significant impact on estimating the burden of illness and can introduce uncertainty in public health decision making.

\section{Conclusions}

In this study, we were able to determine important covariates for natural attack rate of influenza and calculate agespecific and strain-specific attack rates that would serve as essential input parameters to an infectious disease model for influenza. We believe that the use of robust and accurate input parameters are key to reducing uncertainty associated with infectious disease models and therefore would make them more appealing in public health decision making settings.

\section{Abbreviations}

PCR: Polymerase chain reaction; Cl: Confidence interval; ILI: Influenza-like illness; TIV: Trivalent influenza vaccines; LAIV: Live-attenuated influenza vaccines; RCT: Randomized controlled trial; HAl assay: Haemagluttination inhibition assay; MeSH: Medical subject headings; RT-PCR: Reverse transcription polymerase chain reaction.

\section{Competing interests}

ACT (and/or her institution) received funding from the GSK group of companies to complete the work disclosed in this manuscript and declares to have received consulting fees from the GSK group of companies for vaccines studies. CS declares no conflicts of interest. $\mathrm{KJ}$ and $\mathrm{ET}$ are employed by the GSK group of companies. AC was employed by the GSK group of companies at the time of the analysis, and is currently employed at Sanofi Pasteur. AC holds restricted shares in GSK and Sanofi Pasteur.

\section{Authors' contributions}

All authors participated in the design or implementation or analysis, interpretation of the study; and the development of this manuscript. All authors had full access to the data and gave final approval before submission. The work described was carried out in accordance to ICMJE recommendations for conduct, reporting, editing and publications of scholarly work in medical journals. AC and ACT conceived the systematic review. $\mathrm{KJ}, \mathrm{AC}$ and $\mathrm{ET}$ designed the study. CS and ACT performed data collection and prepared data tables. AT and $\mathrm{KJ}$ interpreted the results. $\mathrm{KJ}$ wrote the initial draft of the manuscript.

\section{Acknowledgements}

GlaxoSmithKline Inc. funded this research and was involved in all stages of study conduct, including analysis of the data. GlaxoSmithKline Biologicals SA took in charge all costs associated with the development and publication of this manuscript. We would like to acknowledge Rohita Sharma and Paul Grootendorst with their help with the protocol development for this study. The authors also thank Heather Santiago (publications manager, GSK) and Grégory Leroux (publications manager, Business \& Decision Life Sciences on behalf of GSK Vaccines) for editorial assistance and manuscript coordination and Jemila Hamid for her assistance in producing the figures. ACT acknowledges support from a Canadian Institutes of Health Research/Drug Safety and Effectiveness Network New Investigator Award in Knowledge Synthesis.

\section{Author details}

${ }^{1}$ GSK Inc, 7333 Mississauga Road North, Mississauga, ON L5N 6L4, Canada. ${ }^{2}$ Li Ka Shing Knowledge Institute of St. Michael's Hospital, 30 Bond Street, Toronto, ON M5B 1W8, Canada. ${ }^{3}$ Department of Mathematics \& Statistics, University of Guelph, 50 Stone Road East, Guelph, ON N1G 2W1, Canada. ${ }^{4}$ Division of Epidemiology, Dalla Lana School of Public Health, University of Toronto, 155 College St, Toronto, ON M5T 3M7, Canada. ${ }^{5}$ Present address: Sanofi Pasteur, 1755 Steeles Avenue West, Toronto, ON M2R 3T4, Canada.

Received: 25 April 2014 Accepted: 27 November 2014

Published online: 11 December 2014

\section{References}

1. Eisenberg JN, Brookhart MA, Rice G, Brown M, Colford JM Jr: Disease transmission models for public health decision making: analysis of epidemic and endemic conditions caused by waterborne pathogens. Environ Health Perspect 2002, 110:783-790.

2. Keeling MJ, Rohani P: Modeling Infectious Diseases in Humans and Animals. Princeton University Press; 2007

3. Vynnycky E, White R: An Introduction to Infectious Disease Modelling. Oxford University Press; 2010

4. Molinari NA, Ortega-Sanchez IR, Messonnier ML, Thompson WW, Wortley PM, Weintraub E, Bridges CB: The annual impact of seasonal influenza in the US: measuring disease burden and costs. Vaccine 2007, 25:5086-5096.

5. Lee VJ, Chen MI, Yap J, Ong J, Lim WY, Lin RT, Barr I, Ong JB, Mak TM, Goh LG, Leo YS, Kelly PM, Cook AR: Comparability of different methods for estimating influenza infection rates over a single epidemic wave. Am J Epidemiol 2011, 174:468-478.

6. Turner D, Wailoo A, Nicholson K, Cooper N, Sutton A, Abrams K: Systematic review and economic decision modelling for the prevention and treatment of influenza A and B. Health Technol Assess 2003, 7:iii-xiii, 1

7. Tricco AC, Chit A, Soobiah C, Hallett D, Meier G, Chen MH, Tashkandi M, Bauch $C T$, Loeb M: Comparing influenza vaccine efficacy against mismatched and matched strains: a systematic review and meta-analysis. BMC Med 2013, 11:153

8. Tricco AC, Chit A, Hallett D, Soobiah C, Meier G, Chen M, Tashkandi M, Bauch C, Loeb M: Effect of influenza vaccines against mismatched strains: a systematic review protocol. Syst Rev 2012, 1:35.

9. Tricco AC, Ivers NM, Grimshaw JM, Moher D, Turner L, Galipeau J, Halperin I, Vachon B, Ramsay T, Manns B, Tonelli M, Shojania K: Effectiveness of quality improvement strategies on the management of diabetes: $a$ systematic review and meta-analysis. Lancet 2012, 379:2252-2261. 
10. Allemann $\mathrm{S}$, Houriet $\mathrm{C}$, Diem $\mathrm{P}$, Stettler $\mathrm{C}$ : Self-monitoring of blood glucose in non-insulin treated patients with type 2 diabetes: a systematic review and meta-analysis. Curr Med Res Opin 2009, 25:2903-2913.

11. Reed C, Meltzer MI, Daily Kirley P, Emerson R, Aragon D, Hancock E, Butler L, Baumbach J, Hollick G, Bennett N, Phoutrides E, Laidler M, Finelli L: Progress toward routine estimation of the seasonal burden of influenza in the United States [Abstract]. In $49^{\text {th }}$ Annual Meeting of the Infectious Diseases Society of America, Boston, MA; Abstract \#1122; 2011.

12. WHO: Influenza Fact Sheet [http://www.who.int/mediacentre/factsheets/ fs211/en/]

13. Mossong J, Hens N, Jit M, Beutels P, Auranen K, Mikolajczyk R, Massari M, Salmaso S, Tomba GS, Wallinga J, Heijne J, Sadkowska-Todys M, Rosinska M, Edmunds WJ: Social contacts and mixing patterns relevant to the spread of infectious diseases. PLoS Med 2008, 5:E74.

14. Mathews JD, Chesson JM, McCaw JM, McVernon J: Understanding influenza transmission, immunity and pandemic threats. Influenza Other Respi Viruses 2009, 3:143-149.

15. Osterhaus A, De Pooter D: 101 Questions \& Answers On Influenza. Maarssen: Elsevier; 2009.

16. Hay AJ, Gregory V, Douglas AR, Lin YP: The evolution of human influenza viruses. Philos Trans R Soc Lond B Biol Sci 2001, 356:1861-1870.

17. Petrie JG, Ohmit SE, Johnson E, Cross RT, Monto AS: Efficacy studies of influenza vaccines: effect of end points used and characteristics of vaccine failures. $J$ Infect Dis 2011, 203:1309-1315.

18. Couch R: Vaccines and Related Biological Products Advisory Committee [http://www.fda.gov/ohrms/dockets/ac/07/slides/2007-4282s2_11_files/ frame.htm]

19. Glatman-Freedman A, Portelli I, Jacobs SK, Mathew JI, Slutzman JE, Goldfrank LR, Smith SW: Attack rates assessment of the 2009 pandemic H1N1 influenza A in children and their contacts: a systematic review and meta-analysis. PLoS One 2012, 7:E50228.

20. Talaat KR, Greenberg ME, Lai MH, Hartel GF, Wichems CH, Rockman S, Jeanfreau RJ, Ghosh MR, Kabongo ML, Gittleson C, Karron RA: A single dose of unadjuvanted novel $2009 \mathrm{H} 1 \mathrm{~N} 1$ vaccine is immunogenic and well tolerated in young and elderly adults. J Infect Dis 2010, 202:1327-1337.

21. Vynnycky E, Pitman R, Siddiqui R, Gay N, Edmunds WJ: Estimating the impact of childhood influenza vaccination programmes in England and Wales. Vaccine 2008, 26:5321-5330.

22. Fisman DN, Tuite AR: Estimation of the health impact and cost-effectiveness of influenza vaccination with enhanced effectiveness in Canada. PLoS One 2011, 6:E27420.

doi:10.1186/s12879-014-0670-5

Cite this article as: Jayasundara et al:: Natural attack rate of influenza in unvaccinated children and adults: a meta-regression analysis. BMC Infectious Diseases 2014 14:670.

\section{Submit your next manuscript to BioMed Central and take full advantage of:}

- Convenient online submission

- Thorough peer review

- No space constraints or color figure charges

- Immediate publication on acceptance

- Inclusion in PubMed, CAS, Scopus and Google Scholar

- Research which is freely available for redistribution 